\title{
Checking Plane Wave Approximation for Double Crystal Topography with Curved Samples
}

\author{
B. Jenichen, R. Köhler, and W. Möhling \\ ZIE der AdW der DDR Berlin* \\ Z. Naturforsch. 37a, 405-410 (1982); received December 10, 1981 \\ Dedicated to Prof. G. Hildebrandt on the occasion of his $60^{\text {th }}$ birthday
}

The double crystal diffractometer in $n,-n$ position realizes plane wave conditions only approximately. Nevertheless topographs taken with such arrangements are usually interpreted on the basis of contrast simulations starting with plane waves. Observations of diffraction patterns obtained with curved samples in an n,-n setting have lead us to study the validity of such a treatment. Simulations made for different types of the starting wave actually show that plane waves are adequate even in that critical case. However for a full coincidence between simulation and experiment it proved necessary to account for the residual angular spread within the collimated beam by averaging several plane wave simulations.

In the last years double crystal techniques have become a common tool of $\mathrm{x}$-ray topography. Double crystal topography often gives rather complicated contrast features of the defects of interest. Only by comparison to theoretical results the full information of a topograph can be gained. In most cases that means comparison with contrast simulations. Usually the plane wave approximation is considered appropriate. But within the limits of geometrical optics the curvature of the wavefront incident on the sample is not at all negligible. On the other hand, in calculating the contrast of periodic growth striations it proved sufficient to take into account the angular divergence of the beam incident on the sample by averaging several plane wave simulations computed for different deviations from the Bragg condition [1].

Calculating the image width of a point of the source with the angular acceptance of the collimator by means of geometrical optics might not be applicable because due to diffraction effects an image of that width results in a divergence wider than that calculated on the basis of the dynamical theory. Therefore a plane wave approximation is more appropriate than expected on the basis of geometrical optics.

\footnotetext{
* Akademie der Wissenschaften der DDR, Zentralinstitut für Elektronenphysik, 1086 Berlin, Mohrenstr. 40/41, Postfach 1250.
}

Reprint requests to Dr. W. Möhling, Zentralinstitut für Elektronenphysik, Mohrenstraße 40/41, DDR-1086 Berlin.
A case of special interest in this connection is the curved crystal because a not negligible curvature of the incident wavefront should change the topograph markedly. Incidently we found a fine structure in the narrow reflections of curved crystals (Figure 2). That was the basis for some experiments in order to check whether in that relatively critical case the plane wave approximation is adequate or not.

\section{Experiments}

The observations were made during real structure analysis of oxidized silicon. Rectangular samples $\left(1 \times 2 \mathrm{~cm}^{2}\right)$ cut from oxidized 111 wafers and etched from the back side to remove mechanical damage showed a nearly cylindrical curvature around an axis parallel to the shorter edge (parallel $\langle 112\rangle$ in our case - see Figure 1).

The following pictures were taken with a sample of $345 \mu \mathrm{m}$ over all thickness having approximately $1.33 \mu \mathrm{m}$ frontside oxide. A double crystal arrange-

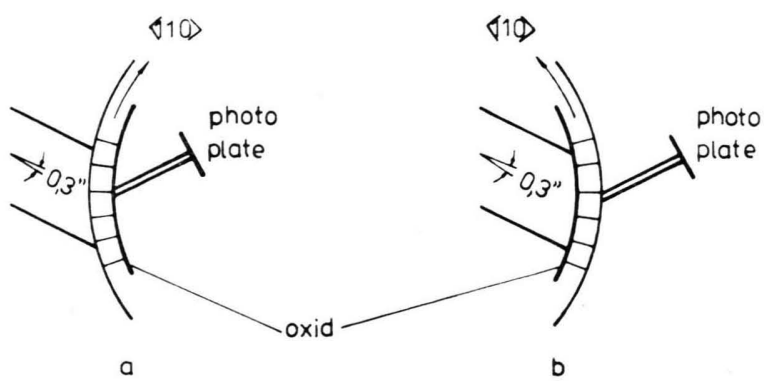

Fig. 1. Diffraction geometries with curved samples. 
ment (Fig. 5) with 440 collimator of asymmetry ratio $b_{\mathrm{c}}=0.18$ for $\mathrm{MoK}_{\alpha 1}$ radiation was applied. The distance between source and collimator was $0.55 \mathrm{~m}$.

Because of the narrow angular spread of the collimated beam the curved sample fullfills the diffraction condition only in a narrow line (Figure 1). However in our experiments we found a fine structure in such reflections resembling a double image at first sight. Figure 2 shows for symmetrical 440 reflection a sequence of 3 reflections taken for 3 different angular positions. Apart from the diffuse appearance of the right one - due to drift during exposure - and some contrast spots due to local defects all images look alike. From the difference in reflection angle and from the linear distance of the reflections recorded successively on the same photoplate a radius of curvature of $11.7 \mathrm{~m}$ is evaluated.

A higher magnification (Fig. 3a) of such a reflection reveals an additional fine structure. This fine structure causes the two weak minima besides the central minimum in the photometer record (Fig. $3 \mathrm{~b})$ along the trace indicated in Fig. $3 \mathrm{a}$.

The foregoing pictures were made with convex curvature of the sample seen from the source, that

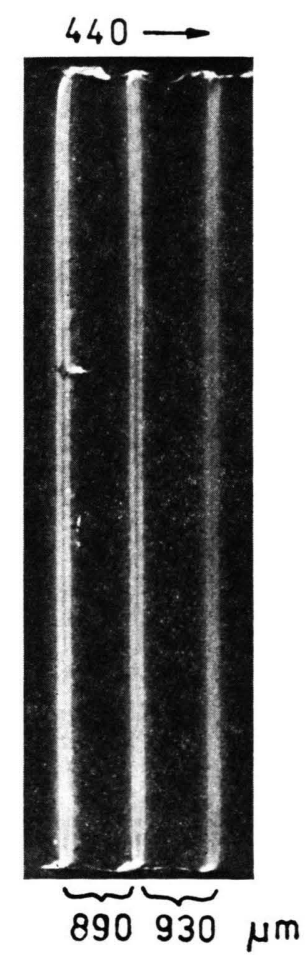

Fig. 2. Three diffraction images taken subsequently on the same photoplate. Between the topographs the curved sample is rotated by $16 \mathrm{sec}$. of arc. Si 440 reflection, MoK $_{\alpha 1}$ geometry as Figure 1 a. means with a geometry according to Figure 1 a. Reflections taken for reversed geometry according to Fig. $1 \mathrm{~b}$ showed in essential the same fine structure (see Figure $4 \mathrm{a}$ ). Unfortunately the details are not brought out so clearly as in the foregoing examples. Obviously this is due to residual mechanical damage having remained on the wafer back side in spite of etching. On the exit surface that damage causes contrast superimposed to the fine structure due to curvature (Figure 4a). Therefore the weak minima in the photometer record of Fig. $3 \mathrm{~b}$ are smoothed out in Fig. $4 \mathrm{~b}$. In the original topograph these minima are indicated nevertheless. If located on the entrance surface, this residual surface damage results in a diffuse scattering in the tails of the reflection only (Figure $3 \mathrm{~b}$ ).

\section{Contrast simulations}

Contrast simulations for different waveforms were made by numerical solution of Takagi's equations $[2,3]$.

Regarding the waveform, a simple model based on geometrical optics seems convincing at first sight. The virtual source distance is increased according to the ratio between accepted and reflected angular divergence $\left(1: b_{c}\right)$ and to the change of the beam diameter $\left(b_{\mathrm{c}}: 1\right)$ (cf. Fig. 5 and 6$)$ [4]:

$$
l_{\text {eff }} \cong \frac{l_{\mathrm{c}}}{b_{\mathrm{c}}^{2}} \quad\left(b_{\mathrm{c}} \ll 1\right) .
$$

$l_{\mathrm{c}}$ is the distance between source and collimator and $b_{\mathrm{c}}$ the asymmetry ratio of the collimator (i.e. cos (angle of incidence)/cos (exit angle); the angles are measured relative to the surface normal).

Then the effective width illuminated on the collimator (projection perpendicular to the direction of the reflected beam, cf. Fig. 6) by one point of the source is given by

$$
w_{\mathrm{G}}=\frac{\omega_{\mathrm{c}}^{0} l_{\mathrm{c}}}{b_{\mathrm{c}}} .
$$

$\omega_{\mathrm{c}}{ }^{0}$ is the halfwidth of the reflection curve of the collimator on the incident side (angular acceptance).

With the data of our experiments $\left(l_{\mathrm{c}}=0.55\right.$, $\left.b_{\mathrm{c}}=0.18\right)$ a virtual source distance of $l_{\mathrm{eff}}=17.2 \mathrm{~m}$ results. Figures $7 \mathrm{a}$ and $\mathrm{b}$ are calculated for an incident spherical wave originating at that distance and correspond to the geometry in Fig. $1 \mathrm{a}$ and b, respectively. These simulations illustrate the formation of 
$440 \rightarrow \quad$ Fig. 3. a) Part of a topograph taken in the geometry of Figure 1a. Si 440 reflection, $\mathrm{MoK}_{\alpha 1}$. Full and broken lines indicate slit and trace, respectively, for the photometer record given in b).

a)

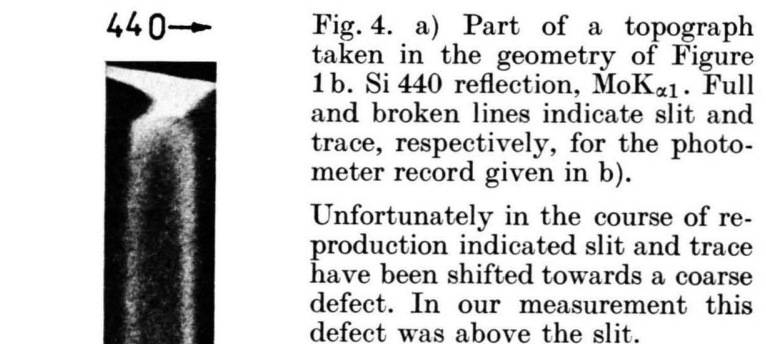

a) defect. In our measurement this defect was above the slit.

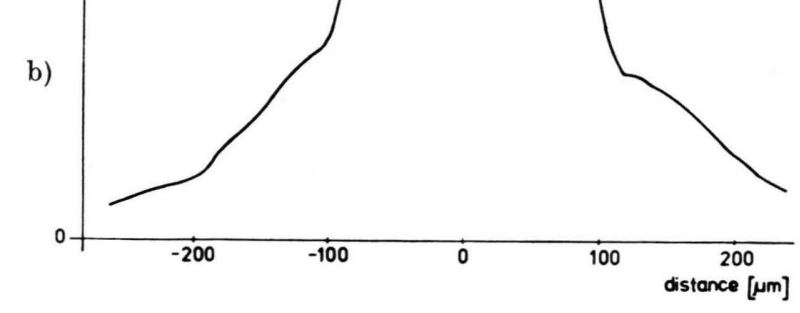

b)

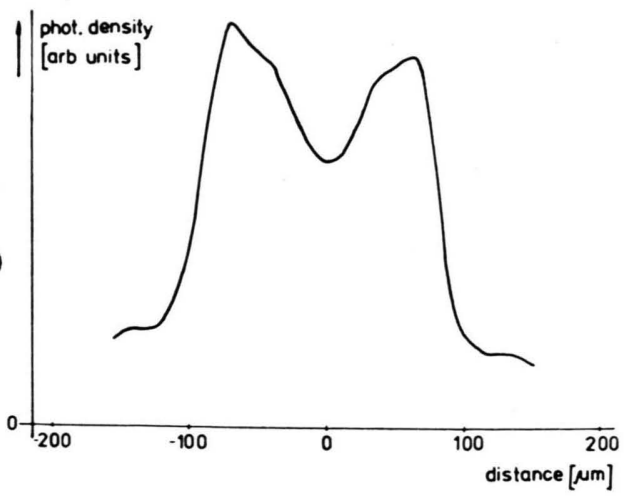




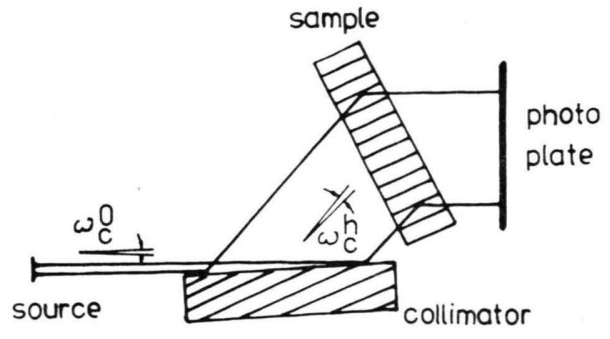

Fig. 5. Double crystal arrangement, $(\mathrm{n},-\mathrm{n})$ setting $\omega_{\mathbf{c}}{ }^{\mathrm{o}}$ : angular acceptance; $\omega_{\mathrm{c}}^{\mathrm{h}}$ : divergence of the reflected beam.

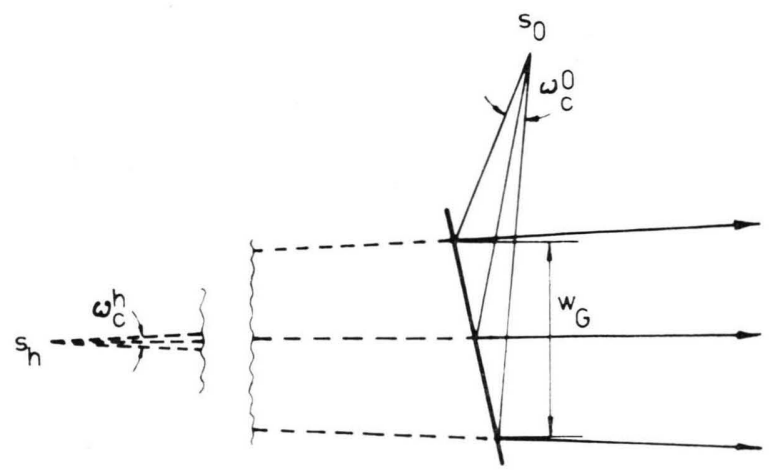

Fig. 6. Virtual source distance as given by geometrical optics $\mathrm{s}_{0}$ : real source point; $\mathrm{s}_{\mathrm{h}}$ : virtual source point.

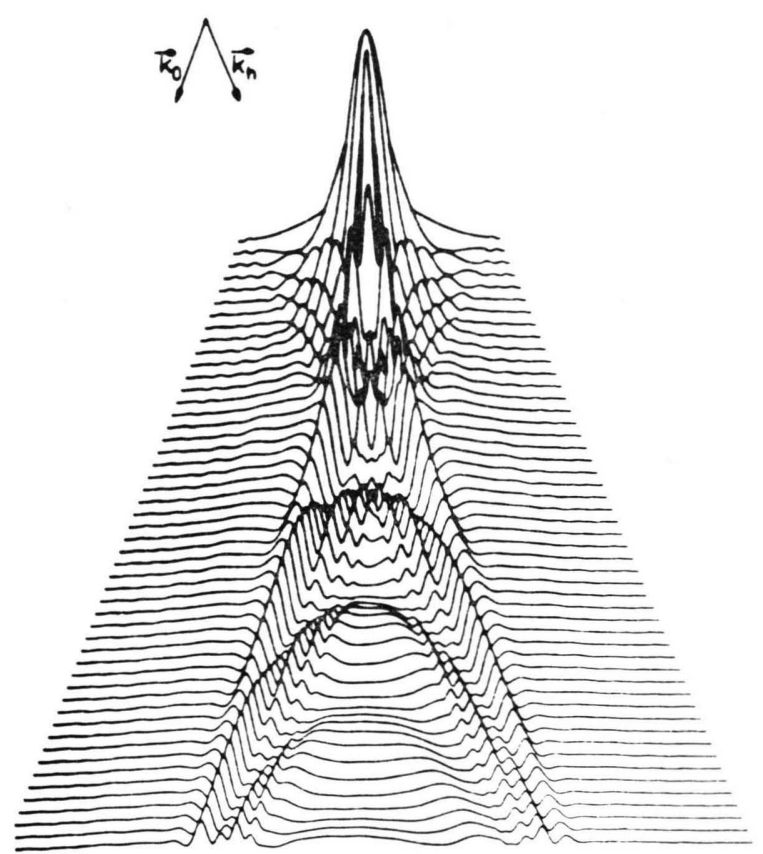

a) contrast with thickness ${ }^{1}$. Obviously there is a striking difference between the two simulations.

On the contrary we obtained no difference for the plane wave case (for negligible absorption - i.e. $\mu \mathrm{t} \lesssim 1$ ). Therefore we present in Fig. 8 a only one example.

The structure of the simulation is partly smoothed by averaging several plane wave simulations in an angular region corresponding to the divergence of the collimated beam in experiment (see Figure $8 \mathrm{~b}$ ). That way of adapting simulations to the experimental situation was already applied in [1].

The simulations are calculated for $\sigma$-polarization only $^{1} \cdot \chi_{\mathrm{h}}=-(122,9+1,44 \mathrm{i}) \cdot 10^{-8}$ and $\mu=14,6$ $\mathrm{cm}^{-1}$ are taken from $[16,17]$,

\section{Discussion}

Interpretation of the full information of $\mathrm{x}$-ray topographs requires the application of well defined

1 The simulations present reflected intensities in a section of a $350 \mu \mathrm{m}$ thick crystal. One of the curves near the bottom (because of uncertainties in thickness and structure factor) should correspond to the photometer records (see Figs. 3, 4).

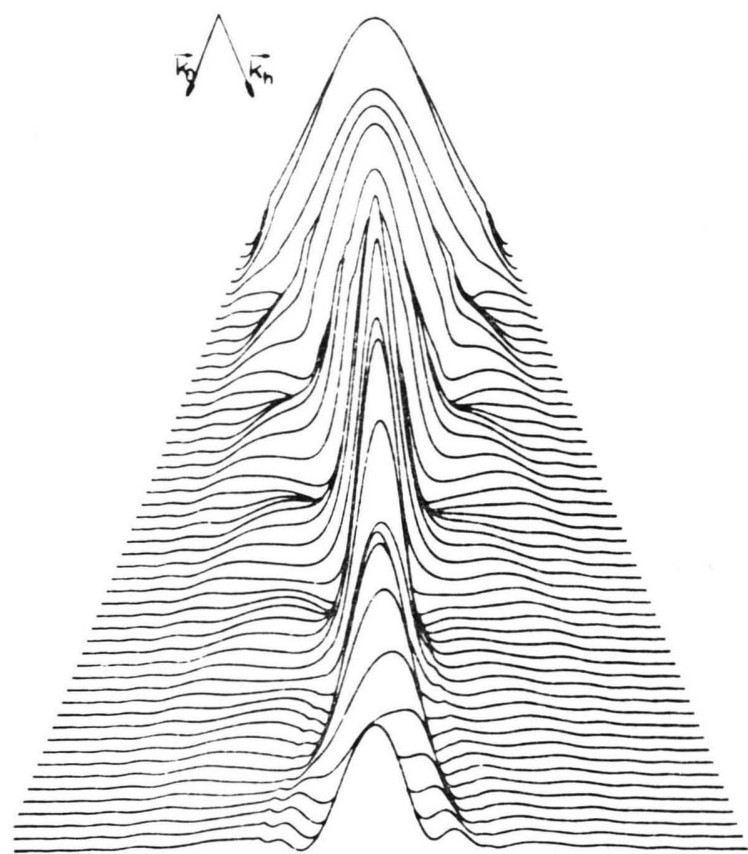

b)

Fig. 7. Contrast simulation for a spherical wave (source distance $17.2 \mathrm{~m}$ ) incident on a sample with $11.7 \mathrm{~m}$ radius of curvature. Si 440 reflection, $M_{0}$, sample thickness: $350 \mu \mathrm{m}$. a), b) Geometries according to Fig. $1 \mathrm{a}$ and b, respectively. 


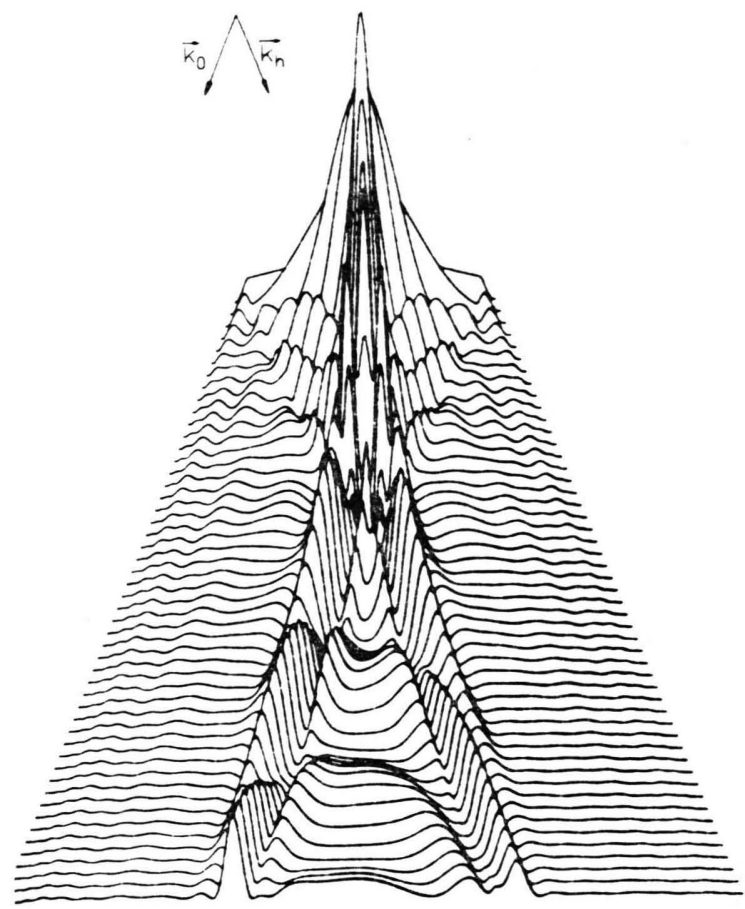

a)

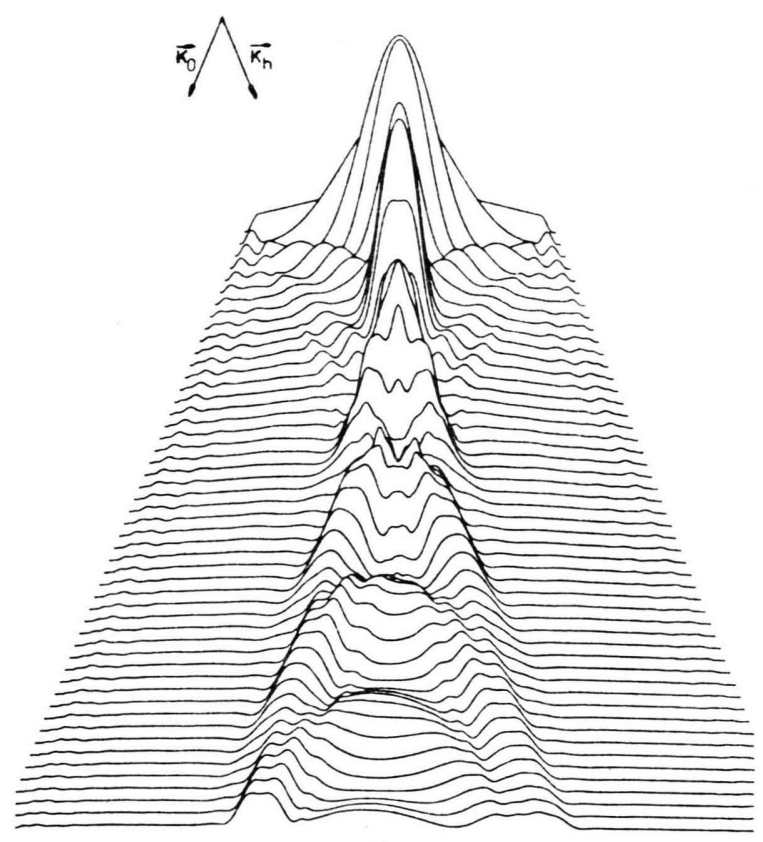

b)

Fig. 8. a) Contrast simulation for a plane wave incident on a sample with $11.7 \mathrm{~m}$ radius of curvature, Si 440 reflection, $\mathrm{MoK}_{\alpha} . \mathrm{b}$ ) average of 6 plane wave simulations as a) shifted one to the next by 0.07 sec. of arc.

incident waves. Therefore an increasing interest in section and plane wave topography is observed. It has been shown that a single source point on the surface might become a poor approximation for the incident wave in section topography. In some cases the final source dimensions [5] as well as the distance between source and crystal [6] have to be taken into account.

Correspondingly an ideal plane incident wave was presumed in most contrast simulations hitherto published for the so called plane wave topography (see e.g. [7-10]). The topographs these contrast simulations are compared to are all taken with the same experimental set-up: the double crystal arrangement (Figure 5). The collimator crystal is asymmetrically cut [11] resulting in an effective divergence of the beam incident to the sample of about $1 / 6$ up to $1 / 3$ of the halfwidth of the reflection curve of the sample crystal.

Presuming an ideal plane wave is obviously in contrast to the calculation of the virtual source distance on the basis of geometrical optics. Some typical values given in table 1 may elucidate that point.

On the other hand the effective image width of a source point in the reflected beam is too small in the case of Mo-radiation. The divergence due to diffraction at a slit of width $w_{\mathrm{G}}$ is of the same order as the divergence of the reflected beam. That means that geometrical optics is insufficient in that case.

In theory there is a correct way to calculate the contrast of a double crystal arrangement. For a single source point the wave reflected at the collimator is calculated and used as the incident wave for the sample crystal. In that way the contrast of some defect is calculated for one source point. Then the intensities evaluated for all source points have to be summed [12]. Calculating the coherence properties follows the same line in principle [13].

Doing numerical contrast simulation in that way means efforts far beyond those necessary in the frame of the plane wave approximation. In a recent paper [1] on the contrast of growth striations we ob- 
Table 1. $\sigma$-polarization only.

\begin{tabular}{llcc}
\hline Radiation & & $\mathrm{MoK}_{\alpha}$ & $\mathrm{CuK}_{\alpha}$ \\
Reflection & & $\mathrm{Si} 440$ & $\mathrm{Si} 220$ \\
Wavelength & pm & 71 & 154 \\
$\omega_{\mathrm{c}}{ }^{*}$ & $\mu \mathrm{rad}$ & 11 & 78 \\
$\omega_{\mathrm{c}}{ }^{\mathrm{h}}$ & $\mu \mathrm{rad}$ & 1.1 & 7.8 \\
$b_{\mathrm{c}}$ & & 0.1 & 0.1 \\
$l_{\mathrm{c}}$ & $\mathrm{m}$ & 0.5 & 0.5 \\
$l_{\mathrm{eff}}$ & $\mathrm{m}$ & 50 & 50 \\
$w_{\mathrm{G}}$ & $\mu \mathrm{m}$ & 55 & 390 \\
$\Delta \omega^{* *}$ & $\mu \mathrm{rad}$ & 1.3 & 0.4 \\
$w_{\mathrm{D}}$ & $\mu \mathrm{m}$ & 31 & 12 \\
\hline
\end{tabular}

* $\omega_{\mathrm{c}}{ }^{\mathrm{h}}$ is the angular divergence of the beam reflected at the collimator.

** $\Delta \omega$ gives the angular width (halfwidth of the main maximum) of a beam cut of a plane wave by a slit of width $w_{\mathrm{G}}$.

tained good results taking into account the divergence of the collimated beam by simply averaging several plane wave simulations. The experimental situation $\left(\mathrm{MoK}_{\alpha 1}\right.$, Si 444 reflection) was similar to that given in column 1 of Table 1 . The positive result of such an approach is not fully explained by the values in Table 1 . On the basis of geometrical optics the coherently illuminated region has a width $w_{G}$. Taking into account the final image width $w_{\mathrm{D}}$ (projection perpendicular to the direction of the reflected beam in the plane of incidence) of a single source point at the surface [14] does not change the situation in principle. But the correspondence between experiment and simulation in this paper and in [1] suggests a coherence length of more than $100 \mu \mathrm{m}$. Presumably even $w_{\mathrm{G}}+w_{\mathrm{D}}$ underestimates the coherence length because of $\Delta \omega>\omega_{\mathrm{c}}{ }^{\mathrm{h}}$. That problem might be settled as it was done for the Laue case in [6].

However even taking for granted that the coherence length suffices for using extended wavefronts without considering coherence explicitly leaves open the question of the appropriate waveform. Plane waves may be well suited in the case of periodic growth striations because their contrast is not very sensitive to some deviation from the plane wave. Yet topographs of a curved crystal should provide a sensitive test for the waveform. Any

[1] B. Jenichen and R. Köhler, phys. stat. sol. (a) 65, 535 (1981).

[2] S. Takagi, J. Phys. Soc. Japan 26, 1239 (1969).

[3] A. Authier, C. Malgrange, and M. Tournarie, Acta Cryst A 24, 126 (1968).

[4] K. Kohra and M. Ando, Nuclear Instrum. Meth. 177, 117 (1980). essential deviation from a plane wave should give different topographs with changing the sign of the curvature. That is illustrated in Figs. $7 \mathrm{a}$ and $\mathrm{b}$ for an incident spherical wave. The origin of the spherical wave is chosen corresponding to the virtual source calculated according to (1).

If the plane wave approximation is applicable, changing the sign of curvature will not change the contrast. Actually the topographs taken for different signs of the curvature are nearly identical (cf. Figs. 3 and 4). This becomes most obvious near the edge of the crystal. Here the thickness decreases due to the etching procedure. The hairpin shaped contrast shows no difference comparing one topograph with the other ${ }^{2}$.

Therefore the plane wave approximation is well suited also in that rather critical case. It should be stressed that a collimator with weak asymmetry was chosen in order to increase the difference in relation to the ideal plane wave case. So the plane wave is an appropriate approximation for double crystal topography at least under experimental conditions similar to those applied here. The real divergence can be accounted for by averaging several plane wave simulations ${ }^{3}$.

According to the table the approximation of geometrical optics (i.e. (1), (2)) is well suited in the case of copper radiation (and the chosen reflection, of course). That means that here the deviation of the waveform from a plane wave should be no longer negligible. But for the case of periodic growth striations the averaging of several plane wave simulations gives correct results too [15]. Nevertheless the values given in Table 1 assure that the distance between collimator and source must not be neglected in a correct treatment of coherence and waveform of the double crystal arrangement.

2 As explained in Chapt. 1 the differences between the topographs and between the photometer records can be attributed to residual surface damage at the back side of the crystal wafer.

3 See [1] and Chapt. 2, compare Fig. $8 \mathrm{a}$ and b with the experimental topographs.

[5] A. Authier, Y. Epelboin, and M. Lefeld-Sosnowska, Communication at the $6^{\text {th }}$ International Summer School on Lattice Defects in Crystals, Krynica 1980.

[6] V. V. Aristov, V. I. Polovinkina, A. H. Afanasev, and V. G. Kohn, Acta Cryst A 36, 1002 (1980).

[7] T. Bedynska, A. Bubakova, and Z. Sourek, phys. stat. sol. (a) 36, 509 (1976). 
[8] J. Gronkowski, phys. stat. sol. (a) 57, 105 (1980).

[9] H. Ishida, N. Miyamoto, and K. Kohra, J. Appl. Cryst. 9, 240 (1976).

[10] K. Ishida, A. Ootuka, and S. Takagi, Phil. Mag. A 43, 935 (1981).

[11] M. Renninger, Z. angew. Phys. 19, 20 (1965).

[12] A. M. Afanasev and V. G. Kohn, Fiz. Tverd. Tela 19, 1775 (1977).
[13] V. Holy, phys. stat. sol. (b) 101, 575 (1980).

[14] T. Uragami, J. Phys. Soc. Japan 27, 147 (1969).

[15] B. Jenichen, Thesis in preparation.

[16] P. A. Doyle and P. S. Turner, Acta Cryst. A 24, 390 (1968).

[17] G. Hildebrandt, J. D. Stephenson, and H. Wagenfeld, Z. Naturforsch. 28 a, 588 (1973). 